\title{
Limiting glioma development by photodynamic therapy-generated macrophage vaccine and allo- stimulation: an in vivo histological study in rats
}

Steen J. Madsen

Catherine Christie

Khoi Huynh

Qian Peng

Francisco A. Uzal

Tatiana B. Krasieva

Henry Hirschberg 


\title{
Limiting glioma development by photodynamic therapy-generated macrophage vaccine and allo-stimulation: an in vivo histological study in rats
}

\author{
Steen J. Madsen, ${ }^{\text {a }}$ Catherine Christie, ${ }^{\mathrm{b}}$ Khoi Huynh, ${ }^{\mathrm{b}}$ Qian Peng, ${ }^{\mathrm{c}}$ Francisco A. Uzal, ${ }^{\mathrm{d}}$ Tatiana B. Krasieva, ${ }^{\mathrm{b}}$ and \\ Henry Hirschberg ${ }^{b, *}$ \\ aUniversity of Nevada, Department of Health Physics and Diagnostic Sciences, Las Vegas, Nevada, United States \\ bUniversity of California, Beckman Laser Institute and Medical Clinic, Irvine, California, United States \\ 'University of Oslo, Pathology Clinic, Rikshospitalet-Radiumhospitalet HF Medical Center, Montebello, Oslo, Norway \\ dUniversity of California, School of Veterinary Medicine, Davis, San Bernardino, California, United States
}

\begin{abstract}
Immunotherapy of brain tumors involves the stimulation of an antitumor immune response. This type of therapy can be targeted specifically to tumor cells thus sparing surrounding normal brain. Due to the presence of the blood-brain barrier, the brain is relatively isolated from the systemic circulation and, as such, the initiation of significant immune responses is more limited than other types of cancers. The purpose of this study was to show that the efficacy of tumor primed antigen presenting macrophage $\left(\mathrm{Ma}^{\mathrm{F} 98}\right)$ vaccines can be increased by: (1) photodynamic therapy (PDT) of the priming tumor cells and (2) intracranial injection of allogeneic glioma cells directly into the tumor site. Experiments were conducted in an in vivo brain tumor development model using Fischer rats and F98 (syngeneic) and $\mathrm{BT}_{4} \mathrm{C}$ (allogeneic) glioma cells. The results showed that immunization with $\mathrm{Ma}$ (acting as antigen-presenting cells), primed with PDT-treated tumor cells $\left(\mathrm{Ma}^{\mathrm{F} 98}\right)$, significantly slowed but did not prevent the growth of F98-induced tumors in the brain. Complete suppression of tumor development was obtained via $\mathrm{Ma}^{\mathrm{F9}}$ inoculation combined with direct intracranial injection of allogeneic glioma cells. No deleterious effects were noted in any of the animals during the 14-day observation period. ๑ 2018 Society of Photo-Optical Instrumentation Engineers (SPIE) [DOI: 10.1117/1.JBO.23.2.028001]
\end{abstract}

Keywords: glioma; immunogenic apoptotic cells; photodynamic therapy-macrophage vaccine; macrophages; photodynamic therapy; allogeneic cells.

Paper 170717PR received Nov. 10, 2017; accepted for publication Jan. 19, 2018; published online Feb. 7, 2018.

\section{Introduction}

Adoptive immunotherapy based on antigen-presenting cells (APCs) vaccine generation for primary CNS tumors has been an active area of research, and the results of several clinical trials using dendritic cell (DC) vaccines have been reported ${ }^{1-5}$ The results of phase I/II trials have suggested efficacy, but it is still undetermined whether immunotherapy can ultimately be beneficial to patients with glioblastoma multiforme (GBM). Further development of APC-based cancer vaccines is required in order to improve efficacy and tolerability.

DCs are immune cells specialized for antigen presentation to naïve T cells, and, as such, they have been used as APCs in the vast majority of immunization studies. Recent work has shown that DCs are part of the mononuclear phagocyte system and that they are indistinguishable from macrophages (Ma) in several key areas including developmental pathways, markers and efficacy as APCs. ${ }^{6}$ Therefore, DCs have no unique adaptation for antigen presentation that is not shared by other $\mathrm{Ma}$, and, as such, it is not surprising that both cell types are equally active vis-a-vis antigen presentation.

Due to the presence of the blood-brain barrier, the brain is relatively isolated from the systemic circulation and therefore, the initiation of significant immune responses is somewhat limited compared to other types of cancers. In addition, the production of immunosuppressive factors and diminished or absent major histocompatibility complex class I expression on glioma cells could limit the effects of immunotherapy. ${ }^{7}$ Nevertheless, Ma and lymphocytes are often found in large numbers in these tumors, clearly indicating that the immune system is actively engaged. ${ }^{8-10}$

Photodynamic therapy (PDT) is a light-based technology that has been used as an adjuvant for the treatment of a variety of cancers including GBM. ${ }^{11-14}$ This localized treatment approach has been proposed as a method to enhance the effectiveness of vaccines for cancer therapy. ${ }^{15-17}$ Although PDT destroys tumor cells directly, a significant immunological component has also been observed. ${ }^{18-21}$ Importantly, for the present work, PDT of glioma cells has been shown to generate multiple antigenic (tumor) peptides, which can be used to activate APCs ex vivo. ${ }^{15}$

The present work is based on the hypothesis that Ma can act as effective APCs in cell-mediated adoptive immunotherapy and the efficacy of the immune response can be increased by ex vivo PDT treatment of the tumor cells, which are used to activate the Ma. In addition, it is hypothesized that the injection of allogeneic tumor cells directly into brain tumors will initiate an immunological rejection reaction, thereby enhancing the specific response in the tumor to the Ma vaccine. This is a proofof-concept study using Ma as APCs and a rat orthotopic glioma 
model in which newly implanted glioma cells are used as a mimic for the infiltrating cells remaining in the resection margin following surgical removal of bulk tumor in patients. The model is an experimental representation of postoperative clinical treatment in which APC vaccination would be initiated at the time of minimal residual disease following surgical resection.

\section{Materials and Methods}

\subsection{Cell Lines}

Rat alveolar Ma (8383) and rat glioma cells (F98) were obtained from the American Type Culture Collection (Manassas, Virginia). The $\mathrm{BT}_{4} \mathrm{C}$ rat glioma cell line was obtained from Oslo University Hospital (Oslo, Norway). The cell line was originally derived from transformed fetal BD-IX rat brain cells after exposure to ethyl-nitrosourea. Both $\mathrm{F} 98$ and $\mathrm{BT}_{4} \mathrm{C}$ cells were grown as monolayers in Dulbecco's modified Eagle medium (Thermo Fisher Scientific, Carlsbad, California) with $10 \%$ fetal bovine serum, $25 \mathrm{mM}$ HEPES buffer $(\mathrm{pH} 7.4)$, penicillin $\left(100 \mathrm{U} \mathrm{ml}^{-1}\right)$, and streptomycin $\left(100 \mu \mathrm{g} \mathrm{ml}^{-1}\right)$ at $37^{\circ} \mathrm{C}$ and $5 \% \mathrm{CO}_{2}$.

\subsection{Effects of Photodynamic Therapy on F98 Cells}

The direct effect of $\mathrm{AlPcS}_{2 \mathrm{a}}$ PDT on F98 glioma cell viability was first assayed in monolayers. For each light level investigated, eight wells (in a 96-well flat bottomed plate) were seeded with F98 at a density of 5000 cells per well and incubated for $24 \mathrm{~h}$. Cells were aliquoted in every third column to reduce light exposure from neighboring wells under illumination. About $1 \mu \mathrm{g} \mathrm{ml}^{-1}$ of $\mathrm{AlPcS}_{2 \mathrm{a}}$ was added to the cells and the incubation was continued for an additional $18 \mathrm{~h}$. The cells were washed twice in fresh medium to remove excess photosensitizer. Light treatment was accomplished with a 670-nm diode laser (Intense, North Brunswick, New Jersey) coupled to a $200-\mu \mathrm{m}$ diameter optical fiber at an irradiance of $5 \mathrm{~mW} \mathrm{~cm}^{-2}$. The cells were irradiated for increasing times resulting in a range of radiant exposures between 0 and $5 \mathrm{~J} \mathrm{~cm}^{-2}$. Following light treatment, incubation was continued for $48 \mathrm{~h}$, at which point the culture medium was replaced with fresh clear buffer containing MTS reagents (Promega, Madison, Wisconsin) and incubated for an additional $2 \mathrm{~h}$. The optical density was measured using an ELx 800uv Universal Microplate Reader (BIO-TEK Instruments, Inc., Winooski, Vermont).

\subsection{Flow Cytometry}

Flow cytometry was used to determine the fraction of viable, apoptotic, and necrotic cells following PDT. Two different fluorescent labels were used: Annexin V-FITC (Beckton, Dickson and Company, Franklin Lakes, New Jersey) to distinguish apoptotic cells, and propidium iodide (PI; Sigma, St. Louis, Missouri) to label necrotic cells. Unlabeled cells were assumed to be viable. The two labels were added to $1 \times 10^{6} \mathrm{~F} 98$ cells with $5 \mu \mathrm{L}$ of Annexin V-FITC and $10 \mu \mathrm{L}$ of $100 \mu \mathrm{g} \mathrm{ml}^{-1}$ PI. The solutions were gently agitated and incubated for $15 \mathrm{~min}$ at room temperature in the dark. For each experiment, a set of control solutions was also prepared. One control remained unlabeled, the second labeled only with Annexin V-FITC, and the third labeled only with PI. Finally, after $15 \mathrm{~min}$ of dark incubation, each tube was analyzed in a flow cytometer (FACS Calibur; Beckton, Dickson and Company) along with CellQuest software.

\subsection{Generation of PDT-Ma Vaccine}

F98 monolayers were grown in T25 flasks until subconfluence and were incubated with photosensitizer $\left(\mathrm{AlPcS}_{2 \mathrm{a}} ; 1 \mu \mathrm{g} \mathrm{ml}^{-1}\right)$ for $18 \mathrm{~h}$. The cells were detached from the flask by enzyme treatment, washed twice to remove excess photosensitizer, and irradiated with $\lambda=670 \mathrm{~nm}$ light at an irradiance and radiant exposure of $5 \mathrm{mWcm}^{-2}$ and $5 \mathrm{~J} \mathrm{~cm}^{-2}$, respectively. The PDTtreated F98 cells $\left(1 \times 10^{6}\right)$ were cocultured with $0.5 \times 10^{6}$ $\mathrm{Ma}$ for $24 \mathrm{~h}$. The antigen activated $\mathrm{Ma}$ are designated $\mathrm{Ma}^{\mathrm{F} 98}$ in the text and figures. To evaluate morphological changes of the Ma after exposure to PDT-treated F98 cells, $2 \times 10^{4} \mathrm{Ma}^{\mathrm{F} 98}$ cells were placed on a glass bottomed imaging dish and incubated for $18 \mathrm{~h}$. Phase contrast microscopy was carried out both on $\mathrm{Ma}^{\mathrm{F} 98}$ and "empty" Ma for comparison.

\subsection{Experimental Animals}

Inbred male Fischer rats weighing about $350 \mathrm{~g}$ were purchased from Simonsen Laboratories, Inc. (Gilroy, California). Animal care and protocols were in accordance with institutional guidelines. Animal holding rooms were maintained at constant temperature and humidity on a 12-h light and dark schedule at an air exchange rate of 18 changes/h. For the surgical procedures, animals were anaesthetized with isoflurane. A postoperative analgesic (buprenorphine: $0.08 \mathrm{mg} \mathrm{kg}^{-1}$ s.c.) was administered following surgery and twice per day for 3 days thereafter. All animals were euthanized at the end of the study or at the first signs of distress. Euthanasia was accomplished via $\mathrm{CO}_{2}$ inhalation.

\subsection{Tumor Cell Injection}

$\mathrm{F} 98$ and $\mathrm{BT}_{4} \mathrm{C}$ cells were injected stereotactically into the brains of Fischer rats, as previously described. ${ }^{22}$ Briefly, anaesthetized rats were fixed in a stereotactic frame. The skin was incised and a $1.0-\mathrm{mm}$ burr hole was made at the following coordinates: $1 \mathrm{~mm}$ posterior to the bregma, $2 \mathrm{~mm}$ to the right of the midline, and at a depth of $2 \mathrm{~mm}$. The injection device consisted of a 30-G blunt cannula connected through a catheter (Hamilton Co., Reno, Nevada) to an infusion pump (Harvard Apparatus, Holliston, Massachusetts). The cannula was fixed in the electrode holder of the stereotactic frame and then vertically introduced into the brain. A total of $10^{4} \mathrm{~F} 98$ and/or $10^{4} \mathrm{BT}_{4} \mathrm{C}$ cells in 20- $\mu$ l PBS were injected into the brain over a period of $2 \mathrm{~min}$. Following injection, the cannula remained in place for $2 \mathrm{~min}$. Closure was done with bone wax and sutures.

\subsection{Experimental Protocol}

Animals were divided into five cohort groups. The basic protocols for these five groups are shown in Fig. 1. Each group consisted of three animals. Since this was a proof-of-principle histological study, with no survival arm, the limited number of animals used was considered sufficient. Group 1: animals were injected with F98 cells only. Group 2: animals were injected with F98 cells in the right hemisphere (syngeneic controls) and $\mathrm{BT}_{4} \mathrm{C}$ cells in the left hemisphere (allogeneic controls). Group 3: animals were injected with a mixture of $\mathrm{F} 98$ and $\mathrm{BT}_{4} \mathrm{C}$ cells in the right hemisphere. Group 4: animals were injected (i.p.) with a combination of PDT-treated F98 tumor cells and $\mathrm{Ma}^{\mathrm{F} 98}$. Forty-eight hours following immunization, F98 cells were injected into the right hemisphere. Group 5: animals were injected (i.p.) as in group 4. Forty-eight hours 

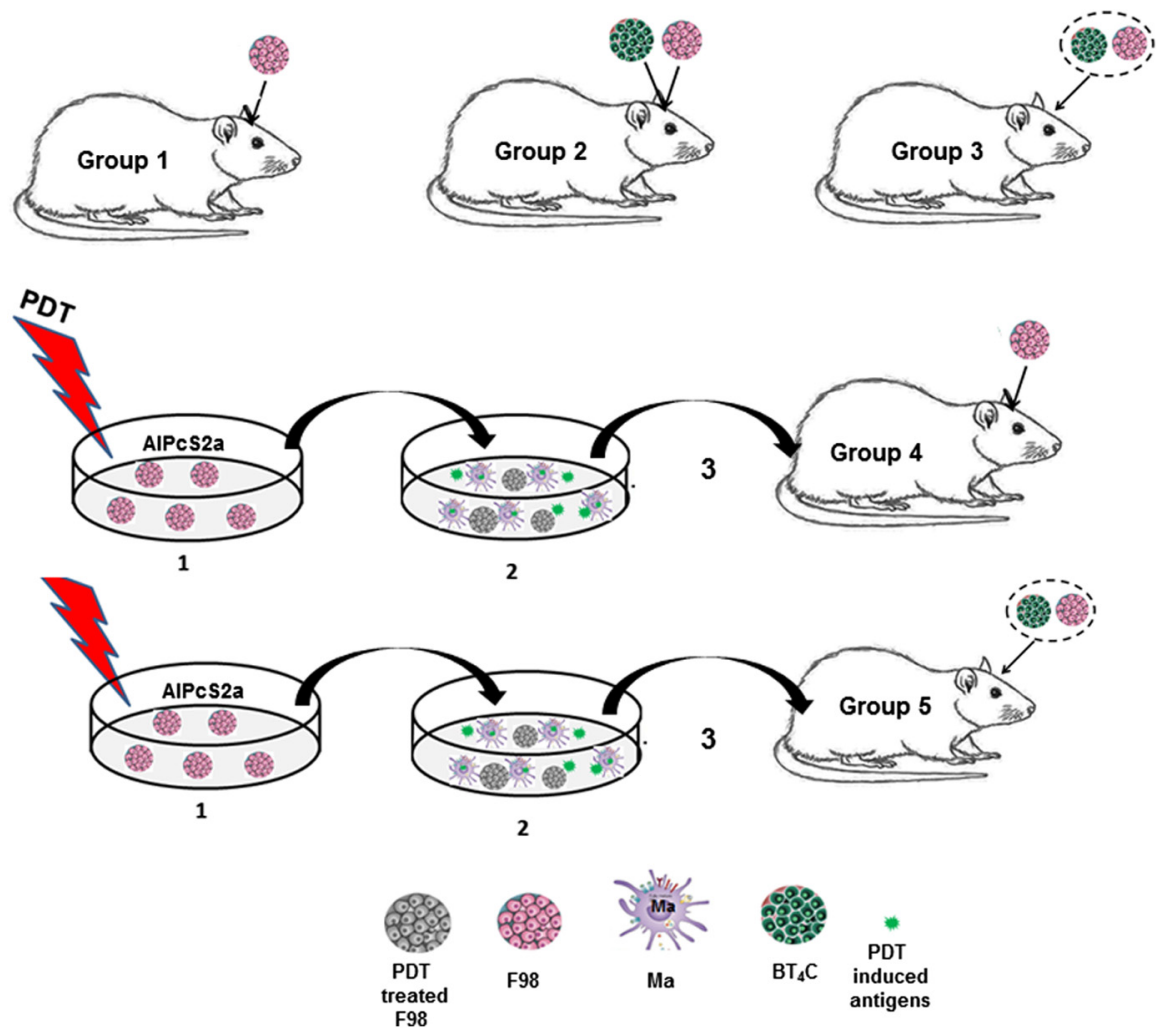

Fig. 1 Experimental protocols for all five cohort groups. Group 1: injected i.c. with F98 cells. Group 2: injected i.c. with $\mathrm{F} 98$ cells in the right hemisphere (syngeneic controls) and $\mathrm{BT}_{4} \mathrm{C}$ cells in the left hemisphere (allogeneic controls). Group 3: injected with a mixture of $\mathrm{F} 98$ and $\mathrm{BT}_{4} \mathrm{C}$ cells in the right hemisphere. Group 4: (1) AlPcS ${ }_{2 a}$ PDT treatment $\left(5 \mathrm{~mW} \mathrm{~cm}^{-2} ; 5 \mathrm{~J} \mathrm{~cm}^{-2}\right)$ of $\mathrm{F} 98$ glioma cells inducing generation of tumor antigens. (2) 24-h coincubation of Ma and PDT-treated F98 cells. (3) i.p. injection of $\mathrm{Ma}^{\mathrm{F} 98}$. Forty-eight hours following immunization, $\mathrm{F} 98$ cells were injected into the right hemisphere. Group 5: $\mathrm{Ma}^{\mathrm{F} 98}$ injected i.p. as in group 4. Forty-eight hours following immunization, a mixture of $\mathrm{F} 98$ and $\mathrm{BT}_{4} \mathrm{C}$ cells were injected into the right hemisphere. All animals were followed for 14 days, euthanized and the brains removed. Histology of removed brains was performed with H\&E staining.

following immunization, a mixture of $\mathrm{F} 98$ and $\mathrm{BT}_{4} \mathrm{C}$ cells were injected into the right hemisphere. The animals were followed for 14 days, euthanized, and the brains removed. Histology of removed brains was performed with hematoxylin and eosin (H\&E) staining.

\section{Results and Discussion}

\subsection{PDT Response of F98 Cells}

The direct effects of $\mathrm{AlPcS}_{2 \mathrm{a}}$ PDT on F98 cell monolayers are shown in Fig. 2(a). At a radiant exposure of $5 \mathrm{~J} \mathrm{~cm}^{-2}$, less than $3 \%$ of the cells were viable. This radiant exposure was therefore used in the subsequent experiments. The results of flow cytometry for two PDT radiant exposures are shown in Fig. 2(b). At $0.5 \mathrm{~J} \mathrm{~cm}^{-2}$, most of the cells were viable as was also seen in the MTS assay [Fig. 2(a)]. At $5 \mathrm{~J} \mathrm{~cm}^{-2}$, only a small fraction of F98 cells were viable as most of the cells died via apoptosis. Figure 2 shows flow plots of apoptosis of untreated (c) or PDTtreated (d) F98 cells stained with annexin V-FITC and PI. Early apoptotic cells were defined as annexin V-FITC+ but PI-. These findings are in good agreement with previous results showing that apoptosis is the primary mode of cell death following low irradiance PDT. ${ }^{23-25}$

Several reports have demonstrated that apoptotic cells are superior to necrotic cells in inducing antitumor immunity. ${ }^{26-30}$
Recent research has led to the concept of immunogenic cell death, which refers to an immunogenic form of apoptosis or necrosis. Cells undergoing immunogenic apoptosis are more potent inducers of antitumor immune responses than cells dying via necrosis. Recent work by Ji et al. ${ }^{31}$ has shown that ALAPDT is capable of generating immunogenic apoptotic cells and that these cells can activate immature DCs. Vaccines using these activated DCs inhibited the growth of squamous cell carcinoma in mice.

\subsection{Morphology of Ma and $\mathrm{Ma}^{\mathrm{Fg}}$}

Coincubation of Ma with PDT-treated F98 cells led to pronounced morphological changes of the $\mathrm{Ma}^{\mathrm{F} 98}$ compared to nonstimulated Ma. As shown in the phase contrast micrographs [Fig. 2(e)], 8383 rat Ma are round, $\sim 10$ to $15 \mu \mathrm{m}$ in diameter and are composed of an equal population of both adherent and floating cells in vitro. By contrast, $\mathrm{Ma}^{\mathrm{F} 98}$ [Fig. 2(f)] were significantly larger and irregular in shape with increased intracellular inclusions. Additionally, the majority of the cells were adherent.

\subsection{Effects of Implanting Syngeneic or Allogeneic Glioma Cells in Nonimmunized Animals}

Control animals implanted with $2 \times 10^{4} \mathrm{~F} 98$ cells into the brain developed large tumors 14 days postinjection (Fig. 3). The lack 
(a)

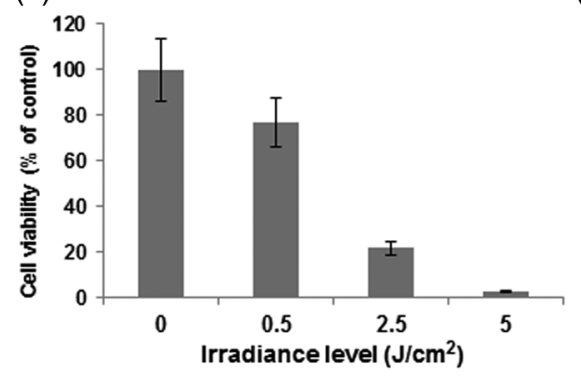

(b)

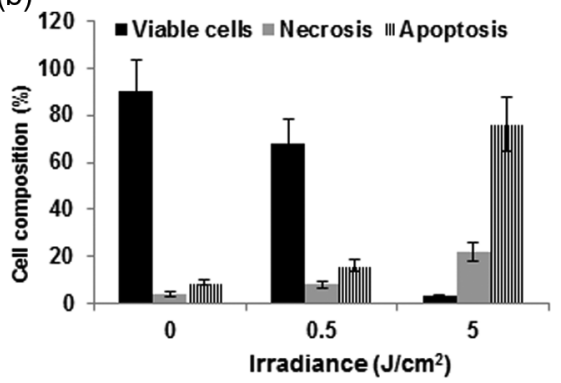

(c)

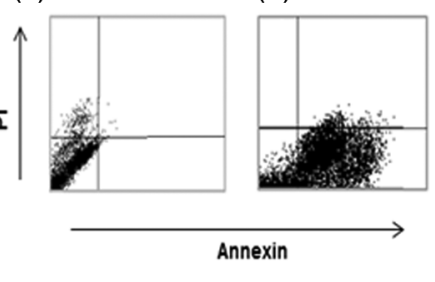

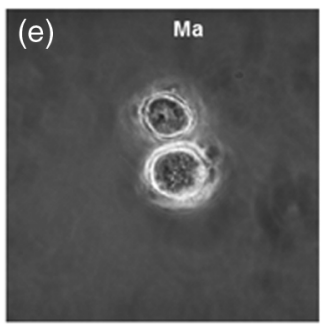

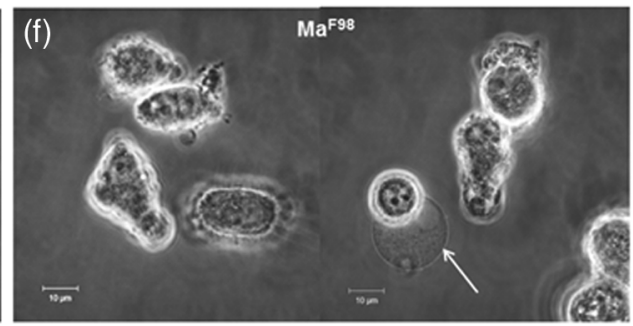

Fig. 2 Generation of immunogenic apoptotic cells and effect on Ma. (a) Cell viability of F98 glioma cells following PDT. (b) Flow cytometry results of PDT-treated F98 cells and flow cytometry scatter plots of (c) nontreated and (d) PDT-treated F98 cells. Cells were incubated in $1 \mu \mathrm{g} \mathrm{ml}^{-1}$ AlPcS $_{2 a}$ for $18 \mathrm{~h}$ and irradiated with 670-nm light at an irradiance of $5 \mathrm{~mW} \mathrm{~cm}^{-2}$. (e) Phase contrast micrograph of nonactivated Ma. (f) Altered Ma morphology following $24 \mathrm{~h}$ coincubation of PDT F98 and Ma (Ma ${ }^{\mathrm{F} 98}$ ). Arrow denotes apoptotic F98 cell and the scale bar represents $10 \mu \mathrm{m}$. PDT experiments were performed in triplicate and error bars denote standard deviations.
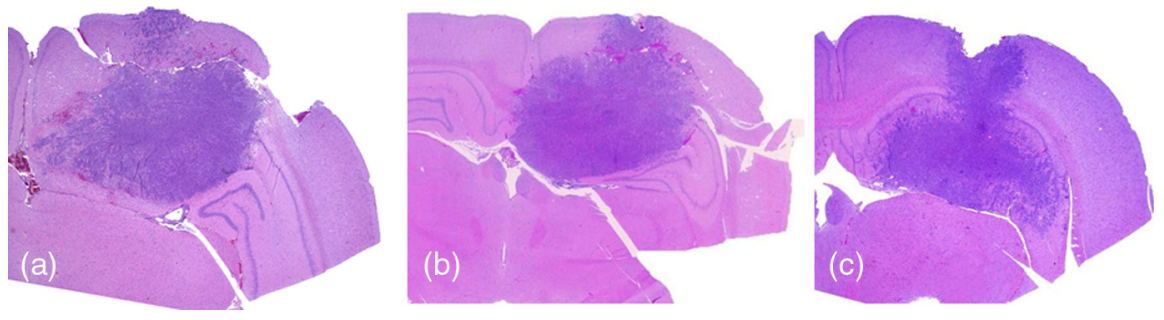

Fig. 3 Photomicrographs of H\&E-stained sections showing F98 cells injected stereotactically into the brains of Fischer rats. (a)-(c) The three animals were euthanized 14 days posttumor implantation.

of encapsulation and significant infiltration of F98 cells into normal brain has been observed previously. ${ }^{32}$ F98 glioma cells have been used in numerous experimental brain tumor studies since the tumors share many fundamental traits with human GBM, including infiltration, rapid growth, extensive neovascularization, absence of encapsulation, and weak immunogenicity. ${ }^{33}$ The weak immunogenicity of the F98 model is a significant
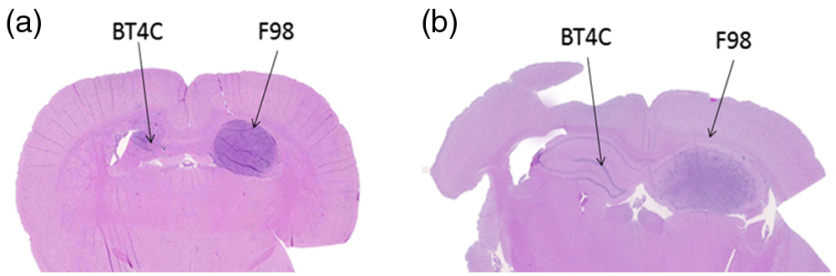

Fig. 4 H\&E-stained sections showing F98 and BT4C cells injected stereotactically into the brains of two Fischer rats ( $a$ and $b)$. All animals developed $\mathrm{F} 98$ tumors while none developed $\mathrm{BT}_{4} \mathrm{C}$ tumors. advantage in evaluating the effects of vaccines compared to other rat glioma models (e.g., C6 and 9L gliosarcoma), which can be highly immunogenic and can therefore evoke intense immune responses on their own.

Group two animals received F98 cells in their right hemisphere and allogeneic $\mathrm{BT}_{4} \mathrm{C}$ cells in their left hemisphere. As illustrated in Fig. 4, all F98-cells developed into tumors while no tumors were observed in the $\mathrm{BT}_{4} \mathrm{C}$-injected hemispheres. This clearly demonstrates the rejection of allogeneic glioma cells in the brain. By contrast, $\mathrm{BT}_{4} \mathrm{C}$ cells readily formed tumors when injected into the brains of syngeneic BD-IX rats. ${ }^{34}$

Interestingly, the syngeneic F98 tumors in these animals were much smaller in comparison to the F98-only tumors implanted in group one animals. Injecting a mixture of F98 and $\mathrm{BT}_{4} \mathrm{C}$ cells into the same hemisphere (group 3 ) also resulted in the development of tumors (Fig. 5) similar in size to those seen in the group 2 animals (Fig. 4). Taken together, these findings indicated that the rejection reaction against the $\mathrm{BT}_{4} \mathrm{C}$ cells could slow tumor progression but was insufficient to completely 
(a)

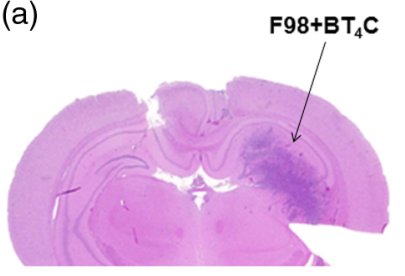

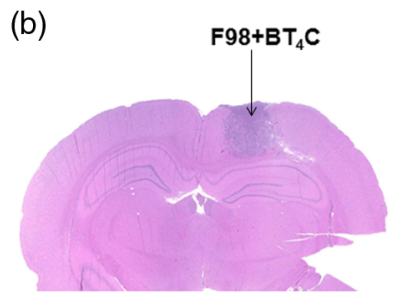

(b)

Fig. $5 \mathrm{H}$ \&E-stained sections showing mixtures of $\mathrm{F} 98$ and $\mathrm{BT}{ }_{4} \mathrm{C}$ cells injected in the same hemisphere of two animals ( $a$ and $b$ ). All animals developed tumors, indicating the rejection reaction against the $\mathrm{BT}_{4} \mathrm{C}$ cells was insufficient to prevent F98 tumor growth.

prevent F98 tumor formation. Although the underlying mechanism of the antitumor immunity observed in these experiments remains to be determined, allogeneic cells likely contain antigen determinants shared with the syngeneic tumor, leading to the observed reduction in tumor growth. This hypothesis is in agreement with the findings of Stathopoulos et al., ${ }^{35}$ who reported that Fischer rats, after initially rejecting subcutaneous C6 allogeneic tumors, failed to develop tumors after subcutaneous injection of syngeneic 9L glioma cells.

\subsection{Tumor Development in Immunized Animals}

Ma were coincubated with PDT-treated apoptotic F98 $\left(\mathrm{Ma}^{\mathrm{F} 98}\right)$ cells for $24 \mathrm{~h}$. A new cohort of animals (Group 4) were inoculated (i.p.) with the resulting mixture of the two cell types. Fortyeight hours following immunization, F98 cells were injected into the right hemisphere. Fourteen days later, the brains were removed and sectioned. The resulting histological sections are shown in Fig. 6. Although tumors developed in all of these $\mathrm{Ma}^{\mathrm{F} 98}$ immunized animals, the tumors were significantly smaller than in the nontreated controls (group 1) as well as in the groups receiving both $\mathrm{F} 98$ and $\mathrm{BT}_{4} \mathrm{C}$ intracranial injection (groups 2 and 3).
In the final cohort (group 5), allogeneic $\mathrm{BT}_{4} \mathrm{C}$ cells were injected along with the F98 glioma cells into $\mathrm{Ma}^{498}$ immunized hosts. As illustrated in Fig. 7, histological sections revealed that there was no evidence of tumor development in any of the animals. Although small remnants of the initial cell inoculate could be seen [Figs. 7(a) and 7(b)], no tumor growth or infiltration was evident. The addition of an allogeneic immune response therefore appeared to enhance the efficacy of the $\mathrm{Ma}^{\mathrm{F} 98}$ vaccine.

High magnification $(250 \times)$ H\&E histological sections of tumors removed from nontreated controls (group 1), F98 + $\mathrm{BT}_{4} \mathrm{C}$ implants (group 3), $\mathrm{Ma}^{\mathrm{F} 98}$ immunized animals (group 4), and $\mathrm{Ma}^{\mathrm{F} 98}$ immunized animals $+\mathrm{BT}_{4} \mathrm{C}$ (group 5) are shown in Figs 8(a)-8(d), respectively. Control tumors were extremely cell rich and compact [Fig. 8(a)], whereas the tumors from the immunized animals demonstrated a much lower cell density [Fig. 8(c)] with areas in the tumor that contained almost no visible tumor cells. Sections from group 5 animals showed only small remnants of the initial cell inoculate, as shown in Fig. 8(d).

The generation of vaccines using PDT has been attempted by a number of groups. For example, Shixiang et al. ${ }^{36}$ generated DC vaccines using PDT-treated C6 glioma cell antigenic peptides. Vaccine efficacy was assessed ex vivo by DC-induced cytotoxic T lymphocyte mediated lysis of C6 target cells. They concluded that PDT of C6 cells significantly enhanced tumor cell immunogenicity compared to freeze-thawed C6 cells.

The use of established allogeneic cell lines to generate glioma vaccines based on HLA typing of individual patients was proposed by Zang et al., ${ }^{37}$ who suggested that HLA typing can predict which combination of cell lines is capable of producing a customized allogeneic vaccine containing the relevant antigens to stimulate the patient's immune system. Alternatively, this approach would allow for autologous DC vaccines to be generated using lysates containing these relevant antigens. Based on previous studies, Stathopoulos et al. ${ }^{38}$ reported a prototype brain cancer vaccine against glioma cells containing multivalent antigens derived from both allogeneic
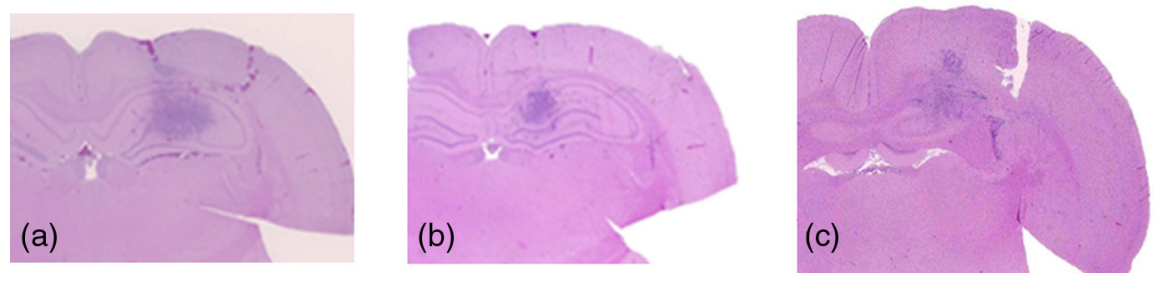

Fig. $6 \mathrm{H} \& \mathrm{E}$-stained sections showing limited tumor development in 3 vaccinated animals $(\mathrm{a}, \mathrm{b}$ and $\mathrm{c})$. Animals were inoculated i.p. with PDT-treated F98 loaded Ma (Ma $\left.{ }^{\mathrm{F9}}\right) 2$ days prior to stereotactic i.c. implantation of $2 \times 10^{4} \mathrm{~F} 98$ tumor cells and sacrificed 14 days later.
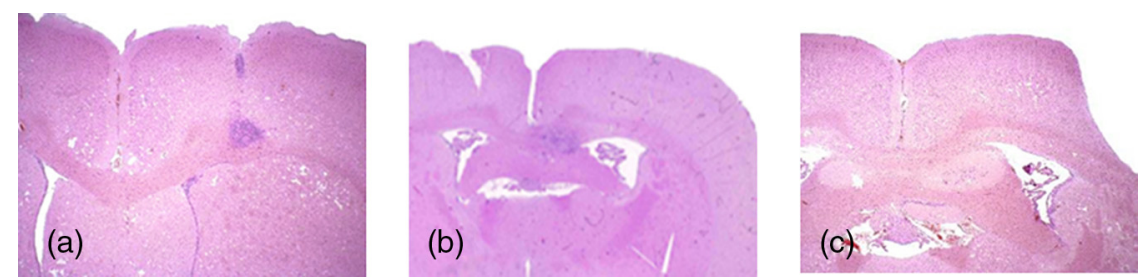

Fig. $7 \mathrm{H \& E}$-stained sections from three animals inoculated i.p. with $\mathrm{Ma}^{\mathrm{F} 98} 2$ days prior to intracranial implantation of $2 \times 10^{4}$ allogeneic $\mathrm{BT}_{4} \mathrm{C}$ cells together with an equal number of syngeneic F98 cells. (a) and (b) Remnants of the implanted cells but no tumor development in any of the animals are shown. (c) No implanted cells are evident. 

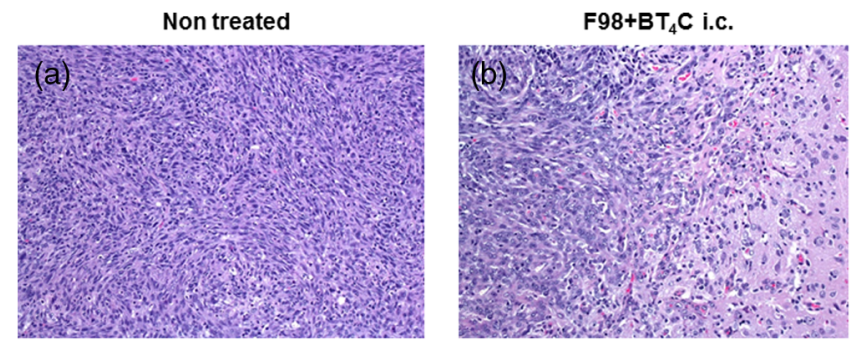

Immunized+F98 i.c.

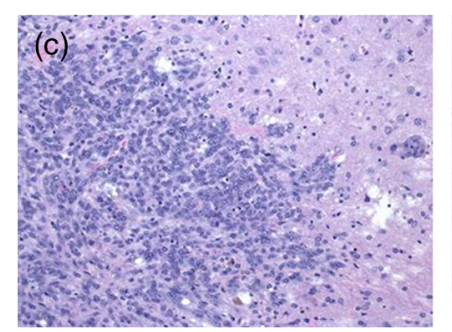

Immunized+F98+BT 4 C i.c

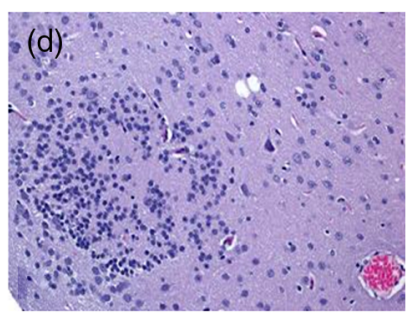

Fig. 8 Magnified (250x) H\&E sections of (a) F98 injected control animals (group 1), (b) F98 + BT4C i.c. (group 3), (c) Ma ${ }^{\mathrm{F} 98}$ vaccine treated animals (group 4), and (d) $\mathrm{Ma}^{\mathrm{F} 98}$ vaccine treated animals + BT4C i.c. (group 5).

and syngeneic cells and lysates. In a subcutaneous tumor model, they demonstrated arrested progression of tumor growth when the vaccine was codelivered with costimulatory agents. The results of a clinical phase I trial on nine recurrent GBM patients have recently been reported. ${ }^{39}$ The immunization approach, consisting of combined administration of multiple allogeneic and autologous tumor-isolated antigens derived from the patient's surgically removed tumor tissue and glioma tumor tissue from three allogeneic donor patients, was based on the preclinical approach described in CNS-1 Lewis rats. ${ }^{38}$ A phase II trial (NCT01903330) employing this approach is currently ongoing.

Although the strategy used in the present proof-of-concept study reported here (i.e., Ma vaccine generated by PDT treatment of the tumor cells combined with direct intracranial alloimmune stimulation) has proven promising, work is in progress aimed at inducing more efficient immunization in a larger number of animals. In the present protocol, the immunizing loaded $\mathrm{Ma}^{\mathrm{F} 98}$ were injected i.p., however, direct injection into the lymph nodes, where $\mathrm{T}$ cell priming occurs, is likely a better approach. Specifically, studies have shown that priming of T cells by APCs in the cervical lymph nodes can induce a homing pattern toward locations within the brain. ${ }^{40}$ Additionally, live allogeneic cells were injected directly into the brain and, as such, this approach is not translatable to the clinic. Developing an Ma vaccine using PDT treatment of both the syngeneic and allogeneic cells, to prime the $\mathrm{Ma}$, as well as a more realistic postoperative brain tumor model are currently being explored.

\section{Conclusions}

The results on the limited number of animals used in the various arms in this pilot study indicated that allogeneic glioma cells injected into the brain did not form tumors but did slow the growth of syngeneic tumors induced in the same animal. Furthermore, although immunization with Ma (acting as APCs), primed with PDT-treated tumor cells ( $\mathrm{Ma}^{\mathrm{F} 98}$ ), significantly slowed the growth of F98-induced tumors in the brain, it failed to prevent tumor formation. Complete suppression of tumor development was obtained by $\mathrm{Ma}^{\mathrm{F} 98}$ inoculation combined with direct intracranial injection of allogeneic cells. No deleterious effects were noted in any of the animals during the 14-day observation period.

\section{Disclosures}

The authors have no relevant financial interests in this article and no potential conflicts of interest to disclose.

\section{Acknowledgments}

The present work is an expansion of studies previously reported in Proceedings of SPIE, vol. 9690, 96900D1-6, 2016. The authors are grateful for support from the Norwegian Radium Hospital Research Foundation. Portions of this work were made possible through access to the LAMMP Program NIBIB P41EB015890. S.J.M. acknowledges the support of the Tony and Renee Marlon Charitable Foundation.

\section{References}

1. H. Ardon et al., "Integration of autologous dendritic cell based immunotherapy in the primary treatment for patients with newly diagnosed glioblastoma multiforme: a pilot study," J. Neuro-oncol. 99, 261-272 (2010).

2. J. S. Yu et al., "Vaccination with tumor lysate pulsed dendritic cells elicits antigen specific, cytotoxic T cells in patients with malignant glioma," Cancer Res. 64, 4973-4979 (2004).

3. R. Yamanaka et al., "Clinical evaluation of dendritic cell vaccination for patients with recurrent glioma: results of a clinical phase I/II trial," Clin. Cancer Res. 11, 4160-4167 (2005).

4. M. J. Hickey et al., "Cellular and vaccine therapeutic approaches for gliomas," J. Transl. Med. 8, 100 (2010).

5. S. Inogés et al., "A phase II trial of autologous dendritic cell vaccination and radio-chemotherapy following fluorescence-guided surgery in newly diagnosed glioblastoma patients," J. Transl. Med. 15, 104 (2017).

6. D. A. Hume, "Macrophages as APC and the dendritic cell myth," J. Immunol. 181, 5829-5835 (2008).

7. M. Weller and A. Fontana, "The failure of current immunotherapy for malignant glioma. Tumor derived TGF- $\beta$, T cell apoptosis, and the immune privilege of the brain," Brain Res. Rev. 21, 128-151 (1995).

8. M. L. Rossi et al., "Immunohistochemical study of mononuclear cell infiltrate in malignant gliomas," Acta Neuropathol. (Berlin) 74, 269277 (1987).

9. M. Shinonaga et al., "Immunohistochemical evaluation of macrophage infiltrates in brain tumors," J. Neurosurg. 68, 259-265 (1988).

10. S. Miescher et al., "In situ characterization, clonogenic potential, and antitumor cytolytic activity of $\mathrm{T}$ lymphocytes infiltrating human brain cancers," J. Neurosurg. 68, 438-448 (1988).

11. S. S. Stylli et al., "Photodynamic therapy of high grade glioma-long term survival," J. Clin. Neurosci. 12, 389-398 (2005).

12. P. J. Muller and B. C. Wilson, "Photodynamic therapy of brain tumor-a work in progress," Laser Surg. Med. 38, 384-389 (2006).

13. H. Stepp et al., "ALA and malignant glioma: fluorescence-guided resection and photodynamic treatment," J. Environ. Pathol. Toxicol. Oncol. 26, 157-164 (2007).

14. M. S. Eljamel, C. Goodman, and H. Moseley, "ALA and photofrin fluorescence-guided resection and repetitive PDT in glioblastoma multiforme: a single centre phase III randomized controlled trial," Lasers Med. Sci. 23, 361-367 (2008).

15. M. Korbelik and J. Sun, "Photodynamic-generated vaccine cancer therapy," Cancer Immunol. Immunother. 55, 900-909 (2006).

16. S. O. Gollnick, L. Vaughan, and B. W. Henderson, "Generation of effective antitumor vaccines using photodynamic therapy," J. Cancer Res. 3(15), 1604-1608 (2002).

17. N. Maeding, T Verwanger, and B. Krammer, "Boosting tumor-specific immunity using PDT," Cancers 8(10), 91 (2016)

18. D. E. Dolmans, D. Fukumura, and R. K. Jain, "Photodynamic therapy for cancer," Nat. Rev. Cancer 3, 380-387 (2003). 
19. A. P. Castano, P. Mroz, and M. R. Hamblin, "Photodynamic therapy and anti-tumour immunity," Nat. Rev. Cancer 6, 535-545 (2006).

20. P. C. Kousis et al., "Photodynamic therapy enhancement of antitumor immunity is regulated by neutrophils," Cancer Res. 67, 10501-10510 (2007).

21. C. M. Brackett and S. O. Gollnick, "Photodynamic therapy enhancement of anti-tumor immunity," Photochem. Photobiol. Sci. 10, 649652 (2011).

22. H. Hirschberg et al., "Minimally invasive photodynamic therapy (PDT) tumor ablation of experimental rat glioma," Minimally Invasive Neurosurg. 49(3), 135-142 (2006).

23. T. M. Sitnik and B. W. Henderson, "The effect of fluence rate on tumor and normal tissue responses to photodynamic therapy," Photochem. Photobiol. 67, 462-466 (1998).

24. S. K. Bisland et al., "Metronomic photodynamic therapy as a new paradigm for photodynamic therapy: rationale and preclinical evaluation of technical feasibility for treating malignant brain tumors," Photochem. Photobiol. 80, 22-30 (2004).

25. M. S. Mathews et al., "Effects of ultra low fluence rate single and repetitive photodynamic therapy on glioma spheroids," Lasers Surg. Med. 41, 578-584 (2009).

26. S. R. Scheffer et al., "Apoptotic, but not necrotic, tumor cell vaccines induce a potent immune response in vivo," Int. J. Cancer 103, 205-211 (2003).

27. R. S. Goldszmid et al., "Dendritic cells charged with apoptotic tumor cells induce long-lived protective CD4+ and CD8+ T cell immunity against B16 melanoma," J. Immunol. 171, 5940-5947 (2003).

28. W. C. Bartholomae et al., "T cell immunity induced by live, necrotic, and apoptotic tumor cells," J. Immunol. 173, 1012-1022 (2004).

29. A. D. Garg et al., "Immunogenic cell death, DAMPs and anticancer therapeutics: an emerging amalgamation," Biochim. Biophys. Acta 1805(1), 53-71 (2010).

30. D. V. Krysko et al., "Immunogenic cell death and DAMPs in cancer therapy," Nat. Rev. Cancer 12(12), 860-875 (2012).
31. J. Ji et al., "Improvement of DC vaccine with ALA-PDT induced immunogenic apoptotic cells for skin squamous cell carcinoma," Oncotarget 6(19), 17135-17146 (2015).

32. S. J. Madsen, K. Kharkhuu, and H. Hirschberg, "Utility of the F98 rat glioma model for photodynamic therapy," J. Environ. Pathol. Toxicol. Oncol. 26(2), 149-155 (2007).

33. R. F. Barth, "Rat brain tumor models in experimental neuro-oncology: the 9L, C6, T9, F98, RG2 (D74), RT-2 and CNS-1 gliomas," J. Neurooncol. 36, 91-102 (1998).

34. E. Angell-Petersen et al., "Influence of light fluence rate on the effects of photodynamic therapy in an orthotopic rat glioma model," J. Neurosurg. 104, 109-117 (2006).

35. A. Stathopoulos et al., "Therapeutic vaccination against malignant gliomas based on allo-recognition and syngeneic tumor antigens: proof of principle in two strains of rat," Vaccine 26, 1764-1772 (2008).

36. Y. Shixiang et al., "Antitumor efficacy of a photodynamic therapy-generated dendritic cell glioma vaccine," Med. Oncol. 28, S453-S461 (2011).

37. J. G. Zhang et al., "Antigenic profiling of glioma cells to generate allogeneic vaccines or dendritic cell based therapeutics," Clin. Cancer Res. 13(2), 566-575 (2017).

38. A. Stathopoulos et al., "Exploring the therapeutic efficacy of glioma vaccines based on allo- and syngeneic antigens and distinct immunological costimulation activators," J. Clin. Cell Immunol. Suppl. 5, 004 (2012).

39. V. E. Schijns et al., "First clinical results of a personalized immunotherapeutic vaccine against recurrent, incompletely resected, treatment-resistant glioblastoma multiforme (GBM) tumors, based on combined allo and auto-immune tumor reactivity," Vaccine 33(23), 2690-2696 (2015).

40. T. Calzascia et al., "Homing phenotypes of tumor-specific CD8 T cells are predetermined at the tumor site by crosspresenting APCs," Immunity 22, 175-184 (2005).

Biographies for the authors are not available. 